\title{
X-ray Characteristics of NGC 3516: A View through the Complex Absorber
}

\author{
T.J.Turner \\ Department of Physics, University of Maryland Baltimore County, Baltimore, MD 21250, U.S.A \\ L.Miller \\ Dept. of Physics, University of Oxford, Denys Wilkinson Building, Keble Road, Oxford OX1 3RH, U.K. \\ S.B.Kraemer \\ Institute for Astrophysics and Computational Sciences, Department of Physics, The Catholic University of \\ America, Washington, DC 20064 \\ and \\ J.N.Reeves \\ Astrophysics Group, School of Physical and Geographical Sciences, Keele University, Keele, Staffordshire \\ ST5 5BG, U.K
}

\begin{abstract}
We consider new Suzaku data for NGC 3516 taken during 2009 along with other recent X-ray observations of the source. The cumulative characteristics of NGC 3516 cannot be explained without invoking changes in the line-of-sight absorption. Contrary to many other well-studied Seyfert galaxies, NGC 3516 does not show a positive lag of hard X-ray photons relative to soft photons over the timescales sampled. In the context of reverberation models for the X-ray lags, the lack of such a signal in NGC 3516 is consistent with flux variations being dominated by absorption changes. The lack of any reverberation signal in such a highly variable source disfavors intrinsic continuum variability in this case. Instead, the colorless flux variations observed at high flux states for NGC 3516 are suggested to be a consequence of Compton-thick clumps of gas crossing the line-of-sight.
\end{abstract}

Subject headings: galaxies: active - galaxies: individual: NGC 3516 - galaxies: Seyfert - X-rays: galaxies

\section{Introduction}

Signatures of ionized gas are commonly observed in Seyfert galaxies. Well-studied sources reveal multiple zones of X-ray absorbing gas covering a range of ionization state, column density, covering fraction and kinematics (e.g. Netzer et al. 2003; Behar et al. 2003; Kaspi et al. 2002; Steenbrugge et al. 2005; Blustin et al. 2005; McKernan et al. 2007; Blustin et al. 2007). The detection of deep K-shell absorption lines from very highly ionized species of Fe (e.g. Reeves et al. 2004; Kaspi et al. 2002; Turner et al. 2008) has shown that X-ray absorbing gas can be spectroscopically traced up to equivalent hydrogen column densities $N_{\mathrm{H}} \sim 10^{24} \mathrm{~cm}^{-2}$. The possible existence of circumnuclear gas at high column densities is of great interest with regard to understanding the mass flow around the black hole and energetic considerations for the system. UV spectroscopy unequivocally shows AGN to possess complex absorption systems and so the presence of a complex 
of X-ray absorbers should be unsurprising.

The systematic spectral hardening exhibited by Seyfert-type AGN as they drop in X-ray flux (e.g. Papadakis et al. 2002; Pounds et al. 2004a,b; Vaughan \& Fabian 2004; Miller et al. 2007, 2008; Turner et al. 2008) can be modeled by changes in covering fraction of X-ray absorbing gas. There are a few cases where changes in individual lines trace rapid changes in the X-ray absorber. For example, an absorption line from Fe XXV detected in NGC 3783 varies on timescales of days (Reeves et al. 2004), likely originating $\sim 0.1 \mathrm{pc}$ from the nucleus. In NGC 1365 variable absorption lines have been detected from Fe XXV and Fe XXVI supporting a picture in which the nucleus suffers variable obscuration by an absorber whose covering fraction changes on short timescales (Risaliti et al. 2005, 2007).

In the broad-line Seyfert 1 galaxy (BLSy1) NGC $3516(\mathrm{z}=0.008836$; Keel 1996), X-ray data covering $0.5-10 \mathrm{keV}$ have revealed a strong signature from a variable X-ray absorbing outflow. Xray grating data from Chandra HETG and XMM RGS show discrete absorption features tracing a range of column densities, ionization states and velocity components for the gas (e.g. Turner et al. 2008; Mehdipour et al. 2010). Here we present new Suzaku data from NGC 3516, taken during 2009 October. We also reconsider recent X-ray observations of this source, seeking to reconcile the spectral and timing behaviour and thus elucidate the true nature of the X-ray signatures of this AGN.

\section{The Suzaku Observations}

Four co-aligned Suzaku (Mitsuda et al. 2007) telescopes focus X-rays on to CCD cameras comprising the X-ray Imaging Spectrometer (XIS Koyama et al. 2007). XIS units 0,2,3 are frontilluminated (FI) while XIS 1 is a back-illuminated CCD. XIS 1 has an enhanced soft-band response but lower area at $6 \mathrm{keV}$ than the FI CCDs as well as a larger background level at high energies. XIS 2 failed on 2006 November 9 and hence was not used. Suzaku also carries a non-imaging, collimated Hard X-ray Detector (HXD, Takahashi et al. 2007), whose PIN detector provides useful AGN data typically over the range $15-70 \mathrm{keV}$.

A Suzaku observation of NGC 3516 was made
2009 Oct (OBSID 704062010) and the data were reduced using v6.9 of HEAsOFT. We screened the XIS and PIN events to exclude data during passage through the South Atlantic Anomaly, starting and ending within 500 seconds of entry or exit. Additionally we excluded data with an Earth elevation angle less than $10^{\circ}$ and cut-off rigidity $>6 \mathrm{GeV}$. The source was observed at the nominal center position for the HXD. FI CCDs were in $3 \times 3$ and $5 \times 5$ edit-modes, with normal clocking mode. We selected good events with grades $0,2,3,4 \& 6$ and removed hot and flickering pixels using the SISCLEAN script. The spaced-row charge injection (SCI) was utilized. The exposure times were $222 \mathrm{ks}$ per XIS unit and $178 \mathrm{ks}$ for the PIN. XIS products were extracted from circular regions of $3.5^{\prime}$ radius while background spectra were extracted from a region of radius 2.5' (offset from the source and avoiding the chip corners, where there are data from the calibration sources).

For the 2009 HXD PIN analysis we used the model "D" background (released 2008 June $17^{1}$ ). The time filter resulting from screening the observational data was applied to the background events model. The ftool HXDPINXBPI was used to create a PIN background spectrum from the screened background data. HXDPINXBPI takes account of the form and flux level of the cosmic X-ray background (Boldt \& Leiter 1987; Gruber et al. 1999) in the Suzaku PIN field of view. We used the response file

ae_hxd_pinxinome3_20090826.rsp for spectral fitting.

During the 2009 Suzaku observation NGC 3516 was found to have source count rates $0.507 \pm 0.001$ (summed XIS 0,3 over $0.75-10 \mathrm{keV}$ ) and $0.049 \pm$ 0.002 (PIN over 15-50 keV) ct/s. The background was $5 \%$ of the total XIS count rate in the full band. The source comprised $9 \%$ of the total counts in the PIN band. The source fluxes for recent observations of NGC 3516 are shown in Table 1.

Spectral fits utilized data from XIS 0 and 3 , in the energy range $0.75-10 \mathrm{keV}$ and from the PIN over $20-50 \mathrm{keV}$. Data in the range $1.75-1.9 \mathrm{keV}$ were also excluded from the XIS spectral analysis owing to uncertainties in calibration around the instrumental Si K edge. We note that data can be

\footnotetext{
${ }^{1}$ http://www.astro.isas.jaxa.jp/suzaku/doc/suzakumemo/ suzakumemo-2007-01.pdf
} 
used down to lower energies, $\sim 0.3 \mathrm{keV}$, for timing analysis. XIS 1 was not used owing to the higher background level at high energies. XIS data were binned at the HWHM resolution for each instrument, optimal for detection of spectral features, while PIN data were binned to be a minimum of $5 \sigma$ above the background level for the spectral fitting. In the spectral analysis, the PIN flux was increased by a factor 1.18 for the new data, appropriate for the cross calibration of instruments for the 2009 observation ${ }^{2}$. The new data were considered in conjunction with those from 2005, whose screening and data reduction are described by Markowitz et al. (2008).

\section{Spectral Fitting Results}

Chandra images of NGC 3516 (George et al. 2002) have shown an extended soft X-ray emission component contributing an observed flux $F_{0.4-2 \mathrm{keV}} \sim 10^{-14} \mathrm{erg} \mathrm{cm}^{-2} \mathrm{~s}^{-1}$ within $\sim 2 \mathrm{kpc}$ (assuming $H_{o}=73 \mathrm{~km} \mathrm{~s}^{-1} \mathrm{kpc}^{-1}$ throughout). In addition, there is a known off-nuclear X-ray source, CXOU 110648.1+723412 (George et al. 2002) that cannot be separated from the nuclear emission using Suzaku. However these two provide only a small fraction of the total soft-band flux for the epochs considered here.

For an immediate visual comparison of data from recent X-ray observations of NGC 3516, we plotted the 2009 XIS spectral data with the 2005 Suzaku observation and other observations made using XMM overlaid (Figure 1). This comparison reveals that during 2009 Suzaku found NGC 3516 in a relatively low flux state. The data show that the line-of-sight absorption is obviously changing between epochs for NGC 3516. Although the hard X-ray flux levels are very similar in the 2005 Suzaku and 2001 Apr XMM data, the former shows a large amount of extra absorption compared to the latter. The lowest level of hard X-ray flux is observed in the new data from 2009, although at this epoch the absorption appears to be also relatively low. It is clear that the source cannot be simply described by a response in ionization of the absorbing gas to changes in the continuum flux.

${ }^{2} \mathrm{ftp} / /$ legacy.gsfc.nasa.gov/suzaku/doc/xrt/suzakumemo2008-06.pdf

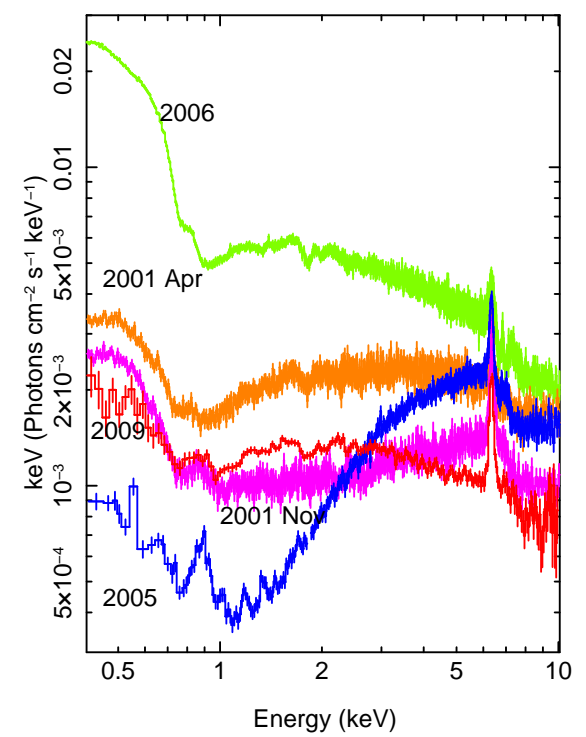

Fig. 1. - The recent X-ray states of NGC 3516 from $X M M$ observations in 2001 and 2006 and from Suzaku observations during 2005 and 2009.

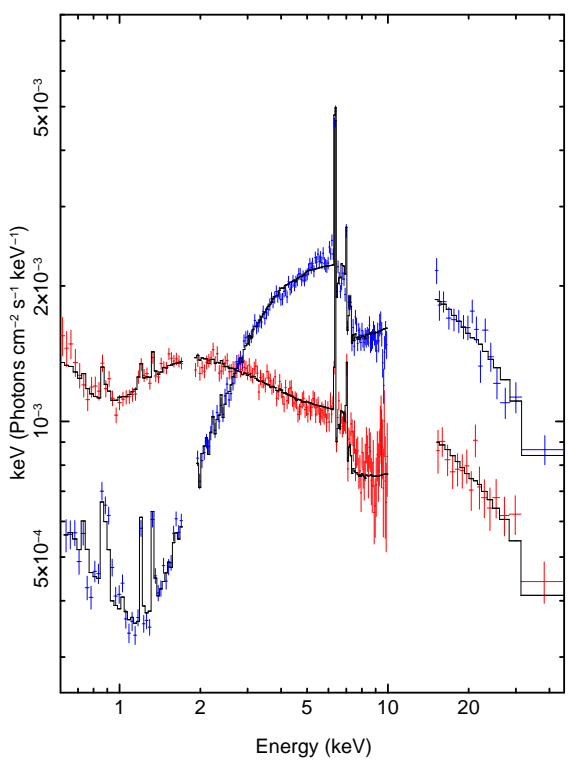

Fig. 2.- Suzaku XIS and PIN data from 2005 (blue) and 2009 (red) along with the absorption model (black solid line). 


\subsection{Fitting Absorption Models}

From detection of discrete absorption lines and from broad spectral curvature, Turner et al. (2008) found evidence for a multi-zoned X-ray absorber in a joint $X M M /$ Chandra campaign during 2006. Markowitz et al. (2008) also found complex absorption to be required to fit the spectrum of NGC 3516 observed by Suzaku during 2005 (and in that spectral model, additionally required a modestly broadened component of Fe K $\alpha$ emission).

We fit the Suzaku 2005 and 2009 data over 0.75$50.0 \mathrm{keV}$ using XSTAR v2.2 to parameterize the ionized gas. The warm absorber table was created with effective micro-turbulence velocity dispersion $\sigma=100 \mathrm{~km} \mathrm{~s}^{-1}$ for gas of density $n=10^{10} \mathrm{~cm}^{-3}$. Here we use the $\xi$ form of ionization parameter, $\xi=L / n r^{2}$, where $L$ is the $1-1000$ Rydberg luminosity and $r$ the absorber-source distance.

The spectral model was constructed with a powerlaw continuum, adding zones of ionized gas until the data were parameterized adequately. The photon index, column densities and ionization states of the gas were allowed to be free but were initially linked to be the same values during 2009 and 2005.

We assumed the gas zones to be layered over each other, with the highest column density zones closest to the nucleus: for this reason, the covering fractions do not total $100 \%$ (Table 2). The model included a full screen of neutral gas fixed at the Galactic line-of-sight column, $3.45 \times 10^{20} \mathrm{~cm}^{-2}$, covering all components and parameterized using the XSPEC model TBABS (Kalberla et al. 2005).

The fit yielded an underlying photon index $\Gamma=$ $2.22 \pm 0.04$ covered by zones of intrinsic absorbing gas (Table 2) having three different covering factors. The outermost layer is Zone 1, comprising low-ionization gas, as established in NGC 3516 from Chandra grating spectroscopy (Turner et al. 2006) and from UV spectroscopy (Kraemer et al. 2002). HETG spectra required two gas components to account for the soft-band absorption features measured during 2006 (Turner et al. 2008). For the joint Suzaku fit we also found that the fit was significantly improved $\left(\Delta \chi^{2}=63\right)$ by allowing the low-ionization zone to have two components (Table 2 Zones $1 \mathrm{a}$ and $1 \mathrm{~b}$, having the same covering fraction). The total column density for Zone 1 is consistent with the sum of the UV absorbing components measured at other epochs (Kraemer et al. 2002).

Additional absorbing zones were found (Table 2) with column densities $\sim 2 \times 10^{23} \mathrm{~cm}^{-2}$ and $\sim 2 \times 10^{24} \mathrm{~cm}^{-2}$, both having $\log \xi \sim 2$. The covering fraction of the Compton-thick zone is around $70 \%$ in 2005 and 2009 spectra. We note that this hard component can, alternatively, be modeled as reflection, as shown by Markowitz et al. (2008).

XSTAR does not calculate the scattered component of X-ray flux from the absorbing gas, however it does calculate the re-emitted line spectrum and we included such a component in the model, placing it behind the Zone 1 absorber complex. With such a placement the re-emission spectrum accounts for the strength of $\mathrm{Fe} \mathrm{K} \alpha$ emission without over-predicting the soft line emission (that is somewhat suppressed by absorption). The ionization parameter of the emitting gas was left free to encompass the possibility that emission arises in a gas component other than one of the absorbers and indeed, the fitted ionization parameter was found to be $\xi=1.53 \pm 0.10$, different to the absorbing gas. The fit gave a reduced $\chi_{r}^{2}=1.24 / 358$ degrees of freedom (d.o.f.). Interestingly, the emission spectrum fitted here is consistent with the ionization-state found for soft-band lines in $X M M$ RGS grating data (Turner et al. 2003).

We stress that the absorption model does not over-predict the $\mathrm{Fe} \mathrm{K} \alpha$ flux, or the flux of any emission line, even if the gas zones are assumed to fully cover the continuum source. The strength of the emission from the absorbing gas depends on the gas geometry, column density and covering/filling factors. Even large column densities for the absorber can result in a small predicted line equivalent width Miller et al. (2009); Yaqoob et al. (2010) when there is expected to be a large opacity at the line energy. It is not suprising that the absorber emission has not been isolated in these data, given the gas geometry suggested in this case.

In this absorption model, the covering fractions of the gas layers varied significantly between epochs (Table 2). Importantly, Zone 2 had a larger covering fraction during $2005(99 \%)$ than during 2009 (86\%), despite the hard-band continuum flux being a factor of 2 higher (Figure 1).

The most highly ionized zone detected at any 
epoch is that traced by absorption lines from Fe XXV and Fe XXVI whose depths indicate an origin in gas having $N_{\mathrm{H}} \sim 3 \times 10^{23} \mathrm{~cm}^{-2}$ and $\log \xi \sim 4.3$ (Turner et al. 2008) These lines were detected in $X M M$ and Chandra data from 2006, and in Suzaku data from 2005, however, they were not detected during the Suzaku observation of 2009, with limits on the equivalents widths of (narrow) Fe XXV and XXVI absorption found to be $<5 \mathrm{eV}$ and $<20 \mathrm{eV}$ respectively.

The absorption modeling and covering fraction changes are fundamentally consistent with the conclusions of Markowitz et al. (2008) and Turner et al. (2008): i.e. the source has a complex absorber and changes in covering by a high column of gas $\sim 10^{23} \mathrm{~cm}^{-2}$ of 'intermediate' ionization (in terms of the X-ray absorbing zones detected in NGC 3516) dominate the spectral variability. Small differences between the fit of Markowitz et al. (2008) and that presented here arise because we are fitting under the joint constraints from 2009 and 2005 epochs, and also because we utilize a new version of XSTAR and use a slightly different model construction.

Constraining the covering fractions of the gas to be the same for 2005 and 2009, allowing only the column densities and ionization states of the layers to be free for each epoch, does not provide a satisfactory joint fit to the two epochs $\left(\chi_{r}^{2}=\right.$ $4.8 / 355$ d.o.f.). It is clear that the source cannot be parameterized satisfactorily without invoking such a mode of variation.

Small improvements to the fit can, of course, be obtained by allowing the absorber column densities and ionization-states to also vary between epochs. However, as Suzaku cannot distinguish which of the layers of gas are varying, we did not find it useful to pursue this line of consideration further.

\subsection{Fe $\mathbf{K} \alpha$ Line Variability}

To test for variability in the $\mathrm{Fe} \mathrm{K} \alpha$ line emission we re-fit the $4.0-7.5 \mathrm{keV}$ data using a simple absorbed power-law model for the local continuum. The line was modeled using a simple Gaussian profile. Initial fits found the line width to be consistent with the HEG value $\sigma=40 \mathrm{eV}$, at all epochs (Turner et al. 2008) and so the fits were rerun with the line width fixed at that value.
The joint 2005/2009 Suzaku fit yielded a peak energy for the line $\mathrm{E}=6.40 \pm 0.06 \mathrm{keV}$. The normalization of the $\mathrm{Fe} \mathrm{K} \alpha$ line was $5.75 \pm 0.24 \times$ $10^{-5}$ photons $\mathrm{cm}^{-2} \mathrm{~s}^{-1}$ (an equivalent width of $164 \mathrm{eV}$ against the observed continuum) during 2005 and $4.16_{-0.32}^{+0.33} \times 10^{-5}$ photons $\mathrm{cm}^{-2} \mathrm{~s}^{-1}$ (equivalent width $253 \mathrm{eV}$ ) during 2009. Thus comparison between the two mean spectra observed using Suzaku reveals strong evidence (> 99.9\% confidence) for variability in $\mathrm{Fe} \mathrm{K} \alpha$ line flux over timescales of years.

To investigate the line behavior on shorter timescales, the $2001 \mathrm{Apr}, 2001$ Nov and 2006 $X M M$ data were re-fit with the new model. Fe K $\alpha$ line fluxes are detailed in Table 3 . In addition to these fits, the individual OBSIDs comprising the $X M M$ data for 2006 were fit individually, yielding Fe K $\alpha$ fluxes sampled on timescales of days: however, no significant variability was detectable on such a timescale (Table 3 ).

\section{Timing Analysis}

We examined the hard $(4.0-7.5 \mathrm{keV})$ and softband (0.3-1 keV) light curves from the Suzaku observations. As was evident from the spectral analysis (Figure 2), the hardness ratio is very different for the two epochs (Figure 3).

The best constraints on the timing properties of NGC 3516 are currently offered by the combined data of 2006. At that epoch the source was bright and, taken together, the $X M M$ and Chandra exposures provide a long baseline of coverage (Turner et al. 2008). We re-constructed the combined $X M M /$ Chandra light curve in selected energy bands (Figure 4). The effective areas and spectral responses of the $X M M$ and Chandra data differ: to create a single light curve, the expected $X M M$ and Chandra count rates in narrow energy bins were calculated from the mean spectral model. Next, the time-dependent observed $X M M$ count rate in those energy bins was rescaled to the expected Chandra count rate using that mean conversion between instruments. The light curve in broader energy bands was then calculated by summing the renormalized values, propagating $X M M$ statistical errors, taking account of the energy-dependent re-normalization. By scaling to the Chandra instrument the uncertainties on the Chandra part of the light curve remain at their 
Poisson values, whereas on the $X M M$ part the uncertainties are slightly worse than Poisson, albeit still much smaller than the Chandra uncertainties. By re-normalizing in fine energy bins, to first-order the effect of spectral variations on the accuracy of the cross-instrument combination are minimized.

For a quantitative assessment of the source variability behavior we follow Miller et al. (2010b,a), in which a maximum-likelihood method is used to measure both the power spectral densities (PSD) and the cross-spectral densities of time series created in a number of broad bands of photon energy. We assume that the physical process(es) generating the count rate variations in the time series is Gaussian (equivalent to the Fourier transforms of the intrinsic time series, before being observationally sampled, having uncorrelated phases). The PSD and cross-spectral density are defined as the Fourier transforms of the autocorrelation and cross-correlation functions. To find the bestfitting PSD and cross-spectra we compute the expected covariance matrix between time samples for an initial set of PSD and cross-spectral values in bins of frequency and iterate to a maximum likelihood solution using the method of Bond, Jaffe, \& Knox (1998). The covariance matrix includes the effects of shot noise. The time lags between bands are evaluated as parameters in the cross-spectral density (Miller et al. 2010b,a).

We applied these methods to the $2006 \mathrm{com}-$ bined XMM/Chandra time series; above $10^{-4} \mathrm{~Hz}$ the $X M M$ data were used alone, owing to the low signal-to-noise ratio for Chandra data sampled at those frequencies. This new evaluation of the PSD (Figure 6) confirms previous conclusions (Edelson \& Nandra 1999) that the PSD shows a steep drop to high frequencies. In contrast to the long XMM/Chandra campaign of 2006, the Suzaku data yielded a relatively short baseline, relatively low amplitude of variability and did not yield conclusive results for the lag spectra. Intriguingly the lag spectra for 2006 data from NGC 3516 showed no positive time lags (Figure 5). At the lowest frequencies sampled there is weak evidence for negative time lags (soft band lagging the hard band) but as the timescales concerned are approaching the longest periods sampled by these observations, caution is required in their interpretation, and independent observations are required to confirm their reality.
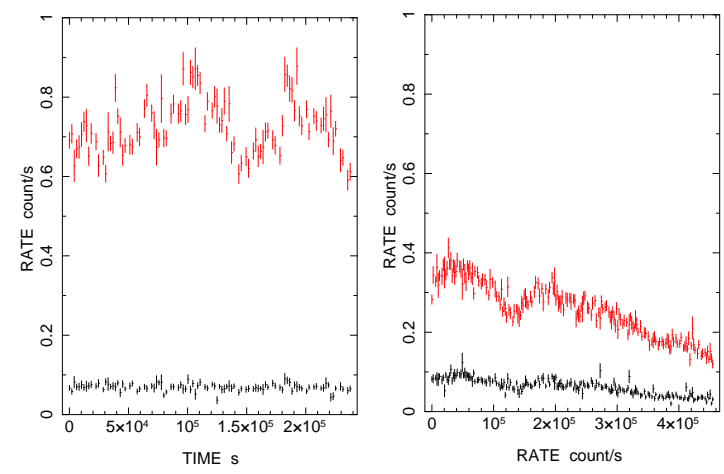

Fig. 3.- Suzaku XIS0 + 3 time series in 2048s bins, from the 2005 and 2009 Suzaku observations. Red points represent $4-7.5 \mathrm{keV}$ and black the $0.3-1 \mathrm{keV}$ band.

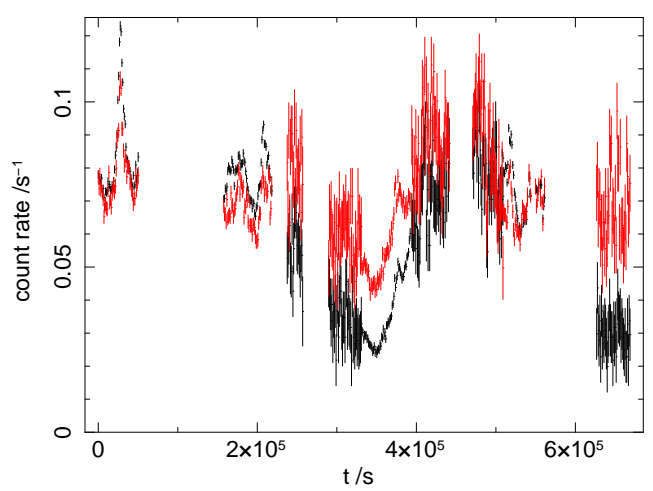

Fig. 4.- The light curve in $2048 \mathrm{~s}$ bins from 2006 XMM pn and Chandra HETG data with soft (black: $0.3-1.0 \mathrm{keV}$ ) and hard bands (red: 4.0$7.5 \mathrm{keV}$ ) overlaid. The $X M M$ light curve rates have been renormalized to the Chandra count rate, and HETG points can be recognized most easily by the relatively large uncertainties on the count rate. 


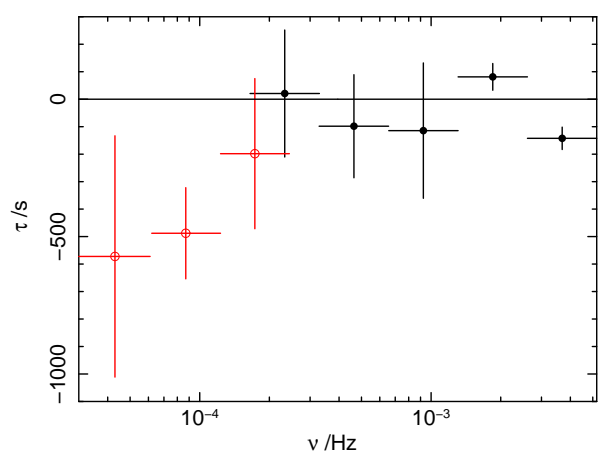

Fig. 5.- Lag spectra for $2006 \mathrm{XMM}$ data, calculated for $4-7.5 \mathrm{keV}$ versus $0.3-1 \mathrm{keV}$. On this plot, positive values of $\tau$ would represent a lag of hard photons relative to the soft.

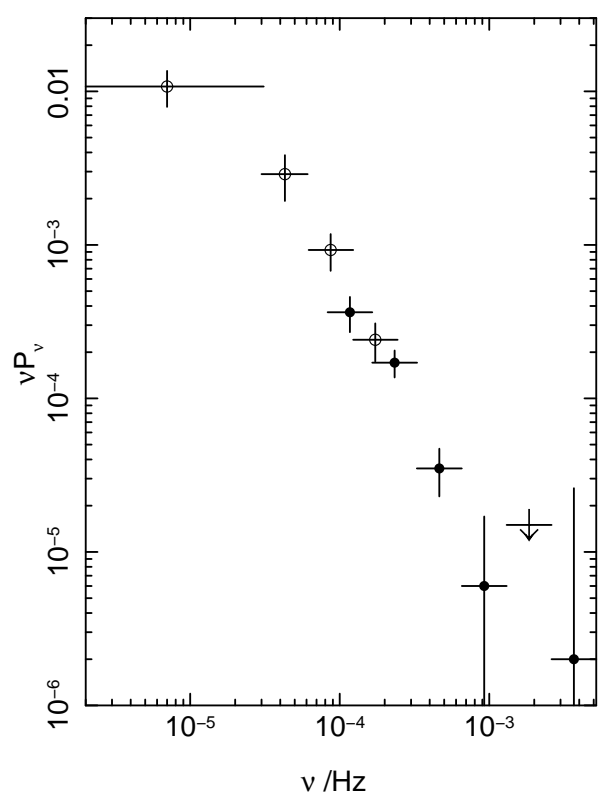

Fig. 6.- PSD from 2006 data over $0.3-1.0 \mathrm{keV}$. The open points denote the combined $X M M$ and Chandra data using 2048 s bins, the solid points denote the $X M M$ data alone, sampled using $96 \mathrm{~s}$ bins.

\section{Discussion}

\subsection{Spectroscopy Results}

Comparison of recent X-ray spectra of NGC 3516 shows that the source has a relatively hard spectrum at some epochs of high X-ray flux. A review of historical X-ray observations of NGC 3516 confirms that there have been numerous epochs during which the source has shown heavy absorption by low-ionization gas, often at high levels of continuum flux (Kruper et al. 1990; Ghosh \& Soundararajaperumal 1991; Kolman et al. 1993; Guainazzi et al. 2001). The covering changes for the absorbing gas are not well-correlated with the observed X-ray continuum flux in NGC 3516, implying that, in addition to the flux variations that are a direct result of obscuration changes, one must invoke either intrinsic continuum variations or 'colorless' variability originating from the passage of Compton-thick blobs of gas across the line-of-sight. Reverberation results favor the latter possibility (see Section 5.2) and in that case the additional component of the model forms a natural extension to the absorber spectral model.

The light-bending model of Miniutti \& Fabian (2004), which attempts to account for flux and spectral variability in AGN by varying the distance of the continuum source perpendicularly from the accretion disk, cannot naturally account for behavior where the source spectrum is found to be hard at a high flux state. Adherence to this model would require an additional variability mechanism to account for all of the X-ray states observed in NGC 3516. Invocation of another source of continuum variability would require an explanation of the lack of positive lags between hard and soft bands, which are observed in other AGN.

Other models that have been suggested to explain the spectral variability of NGC 3516 are also not favored by this comparison of spectral states. Netzer et al. (2002) found ASCA observations of NGC 3516 to be consistent with changes in the ionization state of the X-ray absorber in response to variations in the continuum flux level. Mehdipour et al. (2010) also favor the 'responsive gas' scenario (although based upon a column density an order of magnitude lower than that fitted by Turner et al. 2008, owing to their different modeling of the X-ray continuum and, in particular, 
the inclusion of a black body component that we do not require). However, a responsive-gas model cannot explain the behavior of NGC 3516 across all the X-ray observations; variable-covering absorption is required to explain the overall behavior of the source. In other work, Bhayani \& Nandra (2010) have claimed that differences in the RMS variability between the $4.6-5.0 \mathrm{keV}$ band and 5.0 5.4 and 5.4-5.8 keV bands for the $2001 X M M$ data disfavor an absorption model. However, the predicted measure of RMS variability in a given band depends on the column and ionization-state of the absorbing layer that dominates the variations in that band. As the absorption model matches the X-ray spectrum of NGC 3516 at all flux levels and time slices, it follows de facto that the model reproduces also the RMS spectral variability.

Of course the partial-covering model does not rule out a response of the absorbing gas to changes in the incident flux: it is inevitable (and therefore predicted) that covering changes for inner zones of a complex absorber modify the flux and spectral shape of the continuum reaching zones further out, possibly leading to measurable changes in the ionization balance of the gas.

As discussed for the $2006 X M M /$ Chandra data (Turner et al. 2008), numerous absorption lines are detected in the HETG and RGS gratings, providing a firm basis for the absorption model in NGC 3516. The combined signature of multizoned X-ray absorbers produces curvature in the observed spectral shape, while spectral variability can be explained by changes in covering fraction of the layers. Although current X-ray data do not allow the gas covering fraction to be measured directly from the absorption lines, this quantity can be constrained from the broad X-ray spectral curvature.

This X-ray absorption model comprises a compelling extension of the well-studied UV absorption systems. NGC 3516 shows some of the strongest and most variable intrinsic blueshifted UV absorption lines of any Seyfert 1 galaxy (Kolman et al. 1993; Goad et al. 1999). The C IV absorption is particularly deep and broad and has led some to compare the systems in NGC 3516 to those in broad absorption line QSOs (Ulrich 1988). UV absorption features arise in multiple zones having covering fractions $<1$ (Kraemer et al. 2002). Kraemer et al. (2002) resolve sev- eral kinematic components of absorption in the UV lines. Those authors also isolated zones of gas having signatures spanning both the UV and $\mathrm{X}$-ray regimes, confirming an association between the two bands that had been previously suggested (Guainazzi et al. 2001). Estimates placed the C IV origin at $\sim 10^{16} \mathrm{~cm}$ (Koratkar et al. 1996) with other lines arising out to $\sim 10^{18} \mathrm{~cm}$ from the central source (Kraemer et al. 2002).

Similarly to Seyfert type 2 AGN, NGC 3516 shows a bipolar morphology for the narrow-lineregion gas (Pogge 1989). In NGC 4151, STIS data show evidence for fast-moving clouds existing over a wide angle and originating close to the nucleus (Hutchings et al. 1999; Crenshaw \& Kraemer 2007), possibly collimating the nuclear radiation that excites the NLR gas (Kraemer et al. 2008). A similar model may apply to NGC 3516, where a toroidal distribution of outflowing clouds might collimate the nuclear radiation. Such a toroidal gas distribution could provide X-ray absorption and emission features from different zones within the flow. If our view of NGC 3516 intercepts the edge of the collimating flow we might naturally expect the source behavior to be more variable than sources viewed at face-on or edge-on orientations.

In the context of the partial-covering model, variations of the $\mathrm{Fe} \mathrm{K} \alpha$ flux may arise if the lineemitting region suffers variable obscuration. However, the line flux also depends on the illuminating flux reaching the emitting gas and so the line behavior might be expected to appear complex in a multi-layer absorber model.

HEG data find the Fe $\mathrm{K} \alpha$ emitter to have a bulk velocity consistent with zero (Turner et al. 2008). The HEG constraint on the line width is $25<\sigma<$ $50 \mathrm{eV}$, equivalent to FWHM velocity broadening in the range $3000-6000 \mathrm{~km} / \mathrm{s}$. For gas in a Keplerian orbit the line width corresponds to an origin (417) $\sin ^{2} i$ light-days from the nucleus (where $i$ is the angle of inclination of the orbital plane, assuming a black hole mass $2.95 \times 10^{7} \mathrm{M}_{\odot}$, Nikołajuk et al. 2006).

\subsection{Timing Results}

There has been mounting support for the idea that large amplitude and rapid X-ray flux variations are caused by changes in the line-of-sight absorption (Boller et al. 1997; Guainazzi et al. 
1998; Tanaka et al. 2004). In such a picture, the low/hard states of AGN may be attributable to increases in X-ray obscuration, as suggested for NGC 3516 based on BeppoSAX and $A S C A$ data (Nogami et al. 2004). Deep dips in X-ray flux have been traced by the light curves of MCG-630-15 (McKernan \& Yaqoob 1998) and NGC 3516 (Turner et al. 2008), where data allow us to track the apparent ingress through to the egress of an occultation event. Interestingly, the detailed dip profiles match the profile of dip events in some X-ray binaries, e.g. GRO 1655-40 (Tanaka et al. 2003), where in that case the dip was attributed to an increase in covering fraction of a complex X-ray absorber. Spectral variability in Cir X-1 has also been attributed to changes in covering of a complex absorber (Brandt et al. 1996). The deep dips in NGC 3516 and MCG-6-30-15 are similarly consistent with occultation events. The large number of similarities in observed timing and spectral properties of AGN and stellar binary systems suggests that complex and variable absorption dominates the observed X-ray properties for accreting systems over stellar to galaxy size scales. This, in turn, suggests that the often-outflowing absorber is closely related to the accretion process, and therefore of the highest importance in understanding the fundamental mechanisms in these systems.

Close study of key sources is required to decouple intrinsic continuum variations from absorption effects. The $2006 \mathrm{XMM} /$ Chandra campaign (Turner et al. 2008) showed that in the 0.5-10 keV band, there appear to be two characteristically different modes of X-ray variability: one which results in opacity changes and thus changes in hardness ratio, the other, particularly at high fluxes, which results in no change in hardness ratio. In the context of the complex absorption model, the latter mode may apply when the continuum is uncovered by the fitted absorbers: in the 2006 data this occurred above a flux $F_{2-10} \sim 3 \times 10^{-11} \mathrm{erg} \mathrm{cm}^{-2} \mathrm{~s}^{-1}$. Variability above this threshold was attributed either to intrinsic variations in the continuum or to changes in covering by Compton-thick 'bricks' (Turner et al. 2008).

Recent long X-ray exposures on two bright AGN have allowed significant progress with regard to our general understanding. Suzaku data from NGC 4051 show that the frequency- and energy-dependence of the observed time lags can be explained by the effects of reverberation from a thick shell of material extending to $\sim 600$ gravitational radii from the continuum source (Miller et al. 2010b). That work was the first interpretation of the previously-known positive AGN time lags, that dominate the lag spectra, as arising from reverberation within the X-ray band.

A second important result arises in $X M M$ data from 1H 0707-495. The source shows lags that oscillate between positive and negative values as a function of temporal frequency of variation. However, in 1H 0707-495 it has also been shown (Miller et al. 2010a) that a simple reverberation model can explain the full set of (positive and negative) lags, requiring no additional physical processes to explain the observed lag sign changes.

Many AGN show a lag of hard X-ray photons relative to soft, with the lag increasing to decreasing temporal frequency (e.g. McHardy et al. 2004; Papadakis et al. 2001; Markowitz 2005; Arévalo et al. 2006; Dasgupta \& Rao 2006; Markowitz et al. 2007; Sriram et al. 2009). However, NGC 3516 shows no such positive lag (Figure 5). In the context of our reverberation model for AGN lags (Miller et al. 2010b,a), the lack of a lag in NGC 3516 is interpreted as an absence of a reverberation signal.

These unusual lag results may be associated with the special sight-line at which we observe NGC 3516. The results may be explained if the variations observed during 2006 are attributable predominantly to covering changes of the absorber along our line of sight (i.e. the continuum being intrinsically quasi-constant during those observations), as we would not then expect to see systematic reverberation, which require all lines of sight to vary coherently. Even if the reprocessor subtended a large solid angle to the continuum, there would be no reverberation for the case where there is no intrinsic variation in the continuum. In this case, the PIN-band fluctuations might be attributed to variable obscuration by material having $N_{\mathrm{H}}>10^{25} \mathrm{~cm}^{-2}$.

The tentative negative lag observed at low frequencies in NGC 3516 might be explained by the presence of a reprocessed signal in the soft-band, rather than in the hard band. Such a soft signal would be expected if we observed the emission spectrum from Compton-thin, ionized layers 
of the absorber. This suggestion is supported by the presence of significant soft-band line emission at the lowest X-ray flux states.

\section{Conclusions}

Comparison of 2009 Suzaku data with historic X-ray observations of NGC 3516 shows that relatively hard X-ray spectral forms are exhibited at some epochs of high X-ray flux. Variable X-ray absorption is necessary to explain the range of states observed: simple changes in gas ionization state are insufficient to explain the range of properties exhibited by NGC 3516.

While much of the variability below $10 \mathrm{keV}$ may be explained by changes in the modeled X-ray absorber complex, there is a colorless component of variability evident during high flux states. In past work, the colorless flux variations have been suggested to originate as either intrinsic continuum fluctuations, or to be a consequence of the passage of Compton-thick clumps of gas across the line-of-sight.

NGC 3516 provides a rare example to date of a source showing no significant lag of hard Xray photons relative to soft photons over the timescales studied. This result may be interpreted as an absence of any hard reverberation signal. If this interpretation is correct, despite a large amount of reprocessing material apparently being present around the nucleus, then it seems likely that the continuum source is not intrinsically strongly variable on the timescales studied. We suggest that even the high-state flux variations may be attributed to Compton-thick clumps crossing the line-of-sight. The presence of such high-opacity clumps is a natural extension of the complex absorption model that can describe the X-ray spectrum.

The unusual properties of NGC 3516 may be a consequence of us viewing the source at a rare orientation, where our line-of-sight intercepts the edge of a significant absorber structure that collimates the nuclear radiation leading to the observed biconical structure for the optical narrowline-region gas.

TJT acknowledges NASA grant NNX08AL50G. This research has made use of data obtained from the High Energy Astrophysics Science Archive Re- search Center (HEASARC), provided by NASA's Goddard Space Flight Center.

\section{REFERENCES}

Arévalo, P., Papadakis, I. E., Uttley, P., McHardy, I. M., \& Brinkmann, W. 2006, MNRAS, 372, 401

Behar, E., Rasmussen, A. P., Blustin, A. J., Sako, M., Kahn, S. M., Kaastra, J. S., BranduardiRaymont, G., \& Steenbrugge, K. C. 2003, ApJ, 598,232

Bhayani, S. \& Nandra, K. 2010, MNRAS, 408, 1020

Blustin, A. J., Kriss, G. A., Holczer, T., Behar, E., Kaastra, J. S., Page, M. J., Kaspi, S., Branduardi-Raymont, G., \& Steenbrugge, K. C. 2007, A\&A, 466, 107

Blustin, A. J., Page, M. J., Fuerst, S. V., Branduardi-Raymont, G., \& Ashton, C. E. 2005, A\&A, 431, 111

Boldt, E. \& Leiter, D. 1987, ApJ, 322, L1

Boller, T., Brandt, W. N., Fabian, A. C., \& Fink, H. H. 1997, MNRAS, 289, 393

Bond, J. R., Jaffe, A. H., \& Knox, L. 1998, Phys. Rev. D, 57, 2117

Brandt, W. N., Fabian, A. C., Dotani, T., Nagase, F., Inoue, H., Kotani, T., \& Segawa, Y. 1996, MNRAS, 283, 1071

Crenshaw, D. M. \& Kraemer, S. B. 2007, ApJ, 659,250

Dasgupta, S. \& Rao, A. R. 2006, ApJ, 651, L13

Edelson, R. \& Nandra, K. 1999, ApJ, 514, 682

George, I. M., Turner, T. J., Netzer, H., Kraemer, S. B., Ruiz, J., Chelouche, D., Crenshaw, D. M., Yaqoob, T., Nandra, K., \& Mushotzky, R. F. 2002, ApJ, 571, 265

Ghosh, K. K. \& Soundararajaperumal, S. 1991, ApJ, 383, 574

Goad, M. R., Koratkar, A. P., Kim-Quijano, J., Korista, K. T., O’Brien, P. T., \& Axon, D. J. 1999, ApJ, 524, 707 
Gruber, D. E., Matteson, J. L., Peterson, L. E., \& Jung, G. V. 1999, ApJ, 520, 124

Guainazzi, M., Comastri, A., Stirpe, G. M., Brandt, W. N., Fiore, F., Leighly, K. M., Matt, G., Molendi, S., Puchnarewicz, E. M., Piro, L., \& Siemiginowska, A. 1998, A\&A, 339, 327

Guainazzi, M., Marshall, W., \& Parmar, A. N. 2001, MNRAS, 323, 75

Hutchings, J. B., Crenshaw, D. M., Danks, A. C., Gull, T. R., Kraemer, S. B., Nelson, C. H., Weistrop, D., Kaiser, M. E., \& Joseph, C. L. 1999, AJ, 118, 2101

Kalberla, P. M. W., Burton, W. B., Hartmann, D., Arnal, E. M., Bajaja, E., Morras, R., \& Poeppel, W. G. L. 2005, VizieR Online Data Catalog, 8076, 0

Kaspi, S., et al. 2002, ApJ, 574, 643

Keel, W. C. 1996, AJ, 111, 696

Kolman, M., Halpern, J. P., Martin, C., Awaki, H., \& Koyama, K. 1993, ApJ, 403, 592

Koratkar, A., et al. 1996, ApJ, 470, 378

Koyama, K., et al. 2007, PASJ, 59, 23

Kraemer, S. B., Crenshaw, D. M., George, I. M., Netzer, H., Turner, T. J., \& Gabel, J. R. 2002, ApJ, 577, 98

Kraemer, S. B., Schmitt, H. R., \& Crenshaw, D. M. 2008, ApJ, 679, 1128

Kruper, J. S., Canizares, C. R., \& Urry, C. M. 1990, ApJS, 74, 347

Markowitz, A. 2005, ApJ, 635, 180

Markowitz, A., Papadakis, I., Arévalo, P., Turner, T. J., Miller, L., \& Reeves, J. N. 2007, ApJ, 656,116

Markowitz, A., Reeves, J. N., Miniutti, G., Serlemitsos, P., Kunieda, H., Yaqoob, T., Fabian, A. C., Fukazawa, Y., Mushotzky, R., Okajima, T., Gallo, L. C., Awaki, H., \& Griffiths, R. E. 2008, PASJ, 60, 277

McHardy, I. M., Papadakis, I. E., Uttley, P., Page, M. J., \& Mason, K. O. 2004, MNRAS, 348, 783
McKernan, B. \& Yaqoob, T. 1998, ApJ, 501, L29

McKernan, B., Yaqoob, T., \& Reynolds, C. S. 2007, MNRAS, 379, 1359

Mehdipour, M., Branduardi-Raymont, G., \& Page, M. J. 2010, A\&A, 514, A100

Miller, L., Turner, T. J., \& Reeves, J. N. 2008, A\&A, 483, 437

-. 2009, MNRAS, 399, L69

Miller, L., Turner, T. J., Reeves, J. N., \& Braito, V. 2010a, MNRAS, 408, 1928

Miller, L., Turner, T. J., Reeves, J. N., George, I. M., Kraemer, S. B., \& Wingert, B. 2007, A\&A, 463, 131

Miller, L., Turner, T. J., Reeves, J. N., Lobban, A., Kraemer, S. B., \& Crenshaw, D. M. 2010b, MNRAS, 403, 196

Miniutti, G. \& Fabian, A. C. 2004, MNRAS, 349, 1435

Mitsuda, K., et al. 2007, PASJ, 59, 1

Netzer, H., Chelouche, D., George, I. M., Turner, T. J., Crenshaw, D. M., Kraemer, S. B., \& Nandra, K. 2002, ApJ, 571, 256

Netzer, H. et al. 2003, ApJ, 599, 933

Nikołajuk, M., Czerny, B., Ziółkowski, J., \& Gierliński, M. 2006, MNRAS, 370, 1534

Nogami, K., Negoro, H., Hong, S., \& Mihara, T. 2004, Nuclear Physics B Proceedings Supplements, 132, 209

Papadakis, I. E., Nandra, K., \& Kazanas, D. 2001, ApJ, 554, L133

Papadakis, I. E., Petrucci, P. O., Maraschi, L., McHardy, I. M., Uttley, P., \& Haardt, F. 2002, ApJ, 573, 92

Pogge, R. W. 1989, ApJ, 345, 730

Pounds, K. A., Reeves, J. N., Page, K. L., \& O'Brien, P. T. 2004a, ApJ, 605, 670

—. 2004b, ApJ, 616, 696 
Reeves, J. N., Nandra, K., George, I. M., Pounds, K. A., Turner, T. J., \& Yaqoob, T. 2004, ApJ, 602,648

Risaliti, G., Bianchi, S., Matt, G., Baldi, A., Elvis, M., Fabbiano, G., \& Zezas, A. 2005, ApJ, 630, L129

Risaliti, G., Elvis, M., Fabbiano, G., Baldi, A., Zezas, A., \& Salvati, M. 2007, ApJ, 659, L111

Sriram, K., Agrawal, V. K., \& Rao, A. R. 2009, ApJ, 700, 1042

Steenbrugge, K. C. et al. 2005, A\&A, 434, 569

Takahashi, T. et al. 2007, PASJ, 59, 35

Tanaka, Y., Boller, T., Gallo, L., Keil, R., \& Ueda, Y. 2004, PASJ, 56, L9

Tanaka, Y., Ueda, Y., \& Boller, T. 2003, MNRAS, $338, \mathrm{~L} 1$

Turner, T. J., Kraemer, S. B., Mushotzky, R. F., George, I. M., \& Gabel, J. R. 2003, ApJ, 594, 128

Turner, T. J., Miller, L., George, I. M., \& Reeves, J. N. 2006, A\&A, 445, 59

Turner, T. J., Reeves, J. N., Kraemer, S. B., \& Miller, L. 2008, A\&A, 483, 161

Ulrich, M. 1988, MNRAS, 230, 121

Vaughan, S. \& Fabian, A. C. 2004, MNRAS, 348, 1415

Yaqoob, T., Murphy, K. D., Miller, L., \& Turner, T. J. 2010, MNRAS, 401, 411

This 2-column preprint was prepared with the AAS LATEX macros v5.0. 
TABLE 1

Observed X-RAy Flux

\begin{tabular}{llll}
\hline \hline \multicolumn{1}{c}{ Observation } & Flux (0.5-2) keV & Flux (2-10) keV & Flux (10-50) keV \\
\hline 2001-04 XMM & 0.49 & 2.27 & \\
2001-11 XMM & 0.29 & 1.63 & - \\
2005-10 Suzaku & 0.13 & 2.30 & 8.11 \\
$2006-10$ XMM/Mean & 1.75 & 4.36 & - \\
$2006-10$ XMM/0401 & 2.21 & 5.08 & - \\
$2006-10$ XMM/0501 & 2.02 & 4.49 & - \\
$2006-10$ XMM/0601 & 1.04 & 3.62 & - \\
$2006-10$ XMM/1001 & 1.95 & 4.44 & 3.90 \\
$2009-10$ Suzaku & 0.30 & 1.30 & \\
\hline
\end{tabular}

Note.-The observed flux in erg $\mathrm{cm}^{-2} \mathrm{~s}^{-1}$ 
TABLE 2

Partial Covering Model

\begin{tabular}{lcccc}
\hline \hline zone & $\log \xi$ & $N_{\mathrm{H}}{ }^{1}$ & $C(2005)^{2}$ & $C(2009)^{2}$ \\
\hline $1 \mathrm{a}$ & $0.17 \pm 0.28$ & $0.48_{-0.16}^{+0.18}$ & $99 \pm 1 \%$ & $97 \pm 3 \%$ \\
$1 \mathrm{~b}$ & $0.51_{-0.10}^{+0.25}$ & $1.57_{-0.17}^{+0.64}$ & - & - \\
2 & $2.00_{-0.02}^{+0.01}$ & $19.0_{-0.61}^{+0.48}$ & $99 \pm 1 \%$ & $86 \pm 1 \%$ \\
3 & $2.05 \pm 0.05$ & $200 \pm 6$ & $70 \pm 2 \%$ & $72_{-2}^{+5} \%$ \\
\hline
\end{tabular}

Note.-A column of neutral gas covered all components, representing the Galactic absorption, see text for details. An additional column of ionized gas covered all components during 2005 only, with $N_{\mathrm{H}}=3 \times 10^{23} \mathrm{~cm}^{-2}, \log \xi=4.3$, see text for additional details. Errors are calculated at $90 \%$ confidence. The remaining flux not accounted for in the Table, travels along a line-of-sight that is not covered by the intrinsic absorbers.

${ }^{1}$ Column density in units of $10^{22}$ atom $\mathrm{cm}^{-2}$

${ }^{2}$ Percentage Covering 
TAble 3

Fe K Emission Line

\begin{tabular}{ll}
\hline \hline \multicolumn{1}{c}{ Observation } & \multicolumn{1}{c}{ Normalization $^{1}$} \\
\hline $2001-04 X M M$ & $3.33_{-0.34}^{+0.40}$ \\
$2001-11 X M M$ & $4.02_{-0.23}^{+0.25}$ \\
$2005-10 \mathrm{Suzaku}$ & $5.75 \pm 0.24$ \\
$2006-10 \mathrm{XMM} / \mathrm{Mean}$ & $3.94 \pm 0.17$ \\
$2006-10 X M M / 0401$ & $3.51_{-0.73}^{+0.67}$ \\
$2006-10 \mathrm{XMM} / 0501$ & $4.00_{-1.05}^{+0.61}$ \\
$2006-10 \mathrm{XMM} / 0601$ & $4.25_{-0.83}^{+0.47}$ \\
$2006-10 \mathrm{XMM} / 1001$ & $3.96_{-0.40}^{+0.82}$ \\
$2009-10 \mathrm{Suzaku}$ & $4.16_{-0.32}^{+0.33}$ \\
\hline
\end{tabular}

2009-10 Suzaku

NoTE.-For these fits the line energy was fixed at $6.4 \mathrm{keV}$ and the line width at $\sigma=40 \mathrm{eV}$, as determined from HEG data (Turner et al. 2008) Errors were calculated

${ }^{1}$ Normalization of a Gaussian
odel fit to the Fe K $\alpha$ line, in units $10^{-5}$ photons $\mathrm{cm}^{-2} \mathrm{~s}^{-1}$. 